\title{
ASYMPTOTIC STABILITY RESULTS FOR RETARDED DIFFERENTIAL SYSTEMS
}

\section{K. IGOBI, M. O. EGWURUBE AND M. R. ODEKUNLE}

(Received 24 February 2009; Revision Accepted 6 April 2009)

\section{ABSTRACT}

The transcendental character of the polynomial equation of the retarded differential system makes it difficult to express its solution explicitly. This has cause a set back in the asymptotic stability analysis of the system solutions. Various acceptable mathematical techniques have been used to address the issue. In this paper, the integral-differential equation and the positive symmetric properties of given matrices are used in formulating a Lyapunov functional. The introduction of convex set segment of a symmetric matrix is explored to establish boundedness of the first derivative of the formulated functional. The integral-differential equation is utilized in computing the maximum delay interval for the system to attain stability. Its application to numerical problems confirms the suitability of the test.

KEY WORDS: $\quad$ Asymptotic stability, positive symmetric matrix, convex set segment, integraldifferential equation.

\section{$1.0 \quad$ INTRODUCTION}

The presence of time delay in mathematical model equations has helped in making the system equations more realistic. A retarded differential equation is a functional differential equation with delay (time lag) incorporated only in the state of the system, which accounts for the past state of the system (Asl and Ulsoy, 2003). A general retarded differential equation is of the form,

$$
\dot{x}(t)=f(t, x(t), x(t-n h)), \quad n=1,2,3, ., ., .
$$

where $\dot{x}(t)$ is the derivative of the state function $x(t)$ with respect to time $t$, and $x(t-h)$ is the time lag function, with $h>0$ defining the delay interval.

In recent years, attention has been drawn to dynamic systems whose model equations incorporate time delays, and many results involving such systems have been published (Hale, 1977; Driver, 1977; Hale and Verduyn, 1993; Liu and Mansour, 1984; Svoboda, 2005 and Gugliemi and Hairer, 2007). It is also of note that the survival of any dynamical system depends mostly on the stability of the system solution (Kartsatos, 1980). One criterion for asymptotic stability of a system of differential equation is that all roots of the characteristic equation have negative real parts (Hale, 1977). But the special transcendental character of the polynomial equation of system (1.0) makes it difficult to express the solution explicitly, and hence causes the set back in analyzing the asymptotic stability properties of the system solution.

This set back has been addressed by the extensive use of Lyapunov functional and other acceptable mathematical techniques (Asl and Ulsoy, 2003; Cao et al, 2002; Han, 2001; Hale and Verduyn, 1993 and Driver 1977) in proving conditions for stability of the solution.

Asl and Ulsoy (2003) developed a functional called Lambert function which was used to solve the transcendental characteristic equation of (1.0). Stability properties were analysed based on the negative value of the real part of the characteristic roots. Han (2001) and Cao et al (2002) utilized the linear matrix inequality properties of the system matrix equation, and proved that it satisfies the Lyapunov - Krasovskii conditions for asymptotic stability. Davies (2006) formulated Lyapunov functional using positive definite properties of the matrix equation, and showed that it satisfies the Lyapunov - Razumikin sufficient conditions for asymptotic stability. 
In this paper, the asymptotic stability of the retarded system is addressed by the formulation of Lyapunov functional that satisfies the Lyapunov - Krasovskii conditions for asymptotic stability. The integral-differential equation and the positive symmetric properties of given matrices are used in the formulation of Lyapunov functional. The negative definiteness of the symmetric linear matrix inequality and the boundeness of the first derivative of the formulated functional based on the introduction of a convex set segment of a symmetric matrix is explored to establish asymptotic stability. Also a condition which enhances the computation of the maximum delay interval $\left(h_{\max }\right)$ for (1.0) to assume asymptotic stability is stated using the integral - differential equation of the retarded system.

\section{$1.1 \quad$ Notations}

$P$ is a positive symmetric matrix, $I$ is an $n x n$ identity matrix. $E^{n}$ is the $n$-dimensional Euclidean space, with \|\|$\|$ as the Euclidean vector norm. $B_{H}\left([t-h, t], E^{n}\right)$ is the Banach space of continuously differentiable function on $[t-h, t]$ such that $\left\{h:[t-h, t] \rightarrow E^{n}\right\} . \varphi(s)$ is a continuously differentiable symmetric function with norm in $B_{H}\left([t-h, t], E^{n}\right)$ defined as $\|\varphi(s)\|=\underset{t-h \leq s \leq t .}{\sup } \boldsymbol{t} \varphi(s) \mid$ is a symmetric matrix with diagonal elements $a_{i i}=1$ or 0 , such that each $w_{i}$ defines the $\mathrm{i}^{\text {th }}$ row of $W$, $0 \leq w_{i} \leq 1$, and $\zeta=\left\{x, y \in E^{n} ; w_{i} x-\left(1-w_{i}\right) y\right\} \subset W$ is the convex set segment of $W$.

\subsection{MAIN RESULT}

The aim of this section is to explore the positive symmetric properties of given matrices and the integral - differential equation of retarded system to formulate Lyapunov functional which will satisfy the Lyapunov-Krasovskii conditions for asymptotic stability. These conditions depend on the negative definiteness of a symmetric linear matrix and the upper bound of the first derivative of the formulated functional (Hale, 1977).

Consider an initial value problem of the retarded functional system (1.0) as

$$
\left.\begin{array}{l}
\dot{x}(t)=A x(t)+B x(t-h] \\
x(s)=\varphi(s), \quad t-h \leq s \leq t
\end{array}\right\},
$$

where $\dot{x}(t)$ is the derivative of the state function $x(t)$, and $x(t-h)$ is the delay function with $h>$ 0 as the delay interval. $A, B \in E^{n x n}$ are constant matrices, and $\mathrm{B}$ is symmetric. For a given initial condition $x(s)=\varphi(s), t-h \leq s \leq t$, system (2.1) admits a unique solution (Hale, 1977).

Assume a continuous differentiable symmetric function $\varphi(s) \in B_{H}\left([t-h, t], E^{n}\right)$ as an initial condition of system (2.1). Han (2001) state the integral-differential equation of system (2.1) as

$$
\frac{d}{d t}\left[\varphi(t)-B \int_{t-h}^{t} \varphi(s) d s\right]=(A+B) \quad \varphi(t), t \geq 0 .
$$

The stability analysis of the integral-differential equation $\varphi(t)-B \int_{t-h}^{t} \varphi(s) d s=0$ of (2.2) implies that of (2.1), (Han, 2001).

\section{Theorem 1}

Let there exists a positive symmetric matrix $P \in E^{n \times n}$ which is the unique solution of the quadratic matrix equation $(A+B)^{T} P+P(A+B)=-Q$, where matrix $Q \in E^{n x n}$ and $Q>0$, and a continuous differentiable symmetric function $\varphi(s) \in B_{H}\left([t-h, t] E^{n}\right)$. Given any symmetric positive matrix $\mathrm{W}$ with a convex set segment $\zeta \subset \mathrm{W}$, such that the set

$$
L(\varphi(t))=\left\{\varphi(s) \in B_{H}\left([t-h, t], E^{n}\right] ; V(\varphi(t))=V_{1}(\varphi(t))+V_{2}(\varphi(t)) \text { and } \dot{V}(\varphi(t)) \leq f(\zeta)\right\}
$$

which defines the Lyapunov functional of (2.1), satisfies the symmetric linear matrix inequality (LMI) 


$$
\left[\begin{array}{lll}
\sum_{11} & \sum_{12} & \sum_{13} \\
\sum_{12} & \sum_{22} & \sum_{23} \\
\sum_{13} & \sum_{23} & \sum_{33}
\end{array}\right] \leq 0,
$$

and

$$
\dot{V}(\varphi(t)) \leq \varphi^{T}(t)\left[\begin{array}{lll}
\sum_{11} & \sum_{12} & \sum_{13} \\
\sum_{12} & \sum_{22} & \sum_{23} \\
\sum_{13} & \sum_{23} & \sum_{33}
\end{array}\right] f(\xi) \varphi(t),
$$

then the solution $x(t, \varphi(s))=0$ of $(2.1)$ is asymptotically stable, where $\sum_{11}=\left|-Q+B W^{T} P^{T}\right|, \quad \sum_{22}=|W B|, \quad \sum_{33}=\left|P B(A+B)^{T}\right|$ and $\quad \sum_{i j}=0$, for $i \neq j$.

Proof:

Considering the integral - differential equation of system (2.1) as $\varphi(t)-B \int_{t-h}^{t} \varphi(s) d s$ and any positive symmetric matrix $P$ and $W$, such that $P$

satisfies the quadratic matrix equation $(A+B)^{\top} P+P(A+B)=-Q$, with a continuous differentiable symmetric function $\varphi(s) \in B_{H}\left([t-h, t] E^{n}\right)$, then the Lyapunov functional is defined as $V(\varphi(t))=V_{1}(\varphi(t))+V_{2}(\varphi(t))$, where

$$
\begin{aligned}
& V_{1}(\varphi(t))=\left(\varphi(t)-B \int_{t-h}^{t} \varphi(t) d s\right)^{T} P\left(\varphi(t)-B \int_{t-h}^{t} \varphi(t) d s\right), \\
& V_{2}(\varphi(t))=B \int_{t-h}^{t} \varphi^{T}(s) W \varphi(s) d s .
\end{aligned}
$$

Therefore,

$$
\begin{aligned}
\dot{V}_{1}(\varphi(t)) & =\left(\varphi(t)-B \int_{t-h}^{t} \varphi(s) d s\right)^{T} P \frac{d}{d t}\left(\varphi(t)-B \int_{t-h}^{t} \varphi(s) d s\right) \\
& +\left(\varphi(t)-B \int_{t-h}^{t} \varphi(s) d s\right) P \frac{d}{d t}\left(\varphi(t)-B \int_{t-h}^{t} \varphi(s) d s\right)^{T} \\
& =\left(\varphi(t)-B \int_{t-h}^{t} \varphi(s) d s\right)^{T} P(A+B) \varphi(t)+\left(\varphi(t)-B \int_{t-h}^{t} \varphi(s) d s\right) P((A+B) \varphi(t))^{T} \\
& =\varphi^{T}(t) P(A+B) \varphi(t)+\varphi(t) P(A+B)^{T} \varphi^{T}(t)-B^{T} \int_{t-h}^{t} \varphi^{T}(s) d s P((A+B) \varphi(t)) \\
& -B \int_{t-h}^{t} \varphi(s) d s P\left((A+B)^{T} \varphi^{T}(t)\right) \\
& =\varphi^{T}(t)\left((A+B)^{T} P+P(A+B)\right) \varphi(t)-2 \varphi^{T}(t) P B(A+B)^{T} \int_{t-h}^{t} \varphi(s) d s . \\
V_{2}(\varphi(t)) & =B \int_{t-h}^{t} \varphi^{T}(s) W \varphi(s) d s=B \int_{t-h}^{t} \varphi^{T}(s) W^{T} \varphi(s) d s \\
\dot{V}_{2}(\varphi(t)) & =B \frac{d}{d t}\left(\int_{t-h}^{t} \varphi^{T}(s) W^{T} \varphi(s) d s\right)=B\left[\varphi^{T}(s) W^{T} \varphi(s)\right]_{-h}^{t} \\
& =\varphi^{T}(t) B W^{T} \varphi(t)-\varphi(t-h)^{T} W B \varphi(t-h) .
\end{aligned}
$$

Therefore, $\dot{V}(\varphi(t))=\dot{V}_{1}(\varphi(t))+\dot{V}_{2}(\varphi(t))$,

$$
\begin{aligned}
\dot{V}(\varphi(t))= & \varphi^{T}(t)\left(-Q+B W^{T}\right) \varphi(t) \\
& -\varphi^{T}(t-h) W B \varphi(t-h) \\
& -2 \varphi^{T}(t) P B(A+B)^{T} \int_{t-h}^{t} \varphi(s) d s .
\end{aligned}
$$




$$
=-\sum_{i j}\left(\begin{array}{l}
\varphi(t) \\
\varphi(t-h) \\
\int_{t-h}^{t} \varphi(s) d s
\end{array}\right) \text {, for all } \sum_{\mathrm{ij}}=\left\{\begin{array}{l}
0 i \neq j \\
\text { elsewhere } i=j
\end{array} .\right.
$$

Given any convex set $\zeta=\left\{x, y \in E^{n} ; w_{i} x-\left(1-w_{i}\right) y\right\} \in W, \quad x, y \in E^{n}$

$$
\begin{aligned}
\dot{V}(\varphi(t)) & \leq \varphi^{T}(t)\left(-Q+B\left(w_{i} x+\left(1-w_{i}\right) y\right)^{T} \varphi(t)\right. \\
& -\varphi^{T}(t-h)\left(w_{i} x+\left(1-w_{i}\right) y\right) B \varphi(t-h) \\
& -2 \varphi^{T}(t) P B(A+B)^{T} \int_{t-h}^{t} \varphi(s) d s .
\end{aligned}
$$

Equation (2.5) shows the negative definiteness of the symmetric linear matrix inequality and (2.6) shows the boundedness of the functional, and hence the asymptotic stability of the solution.

\section{Theorem 2}

System (2.1) is asymptotically stable if for any real number $\delta>0$, the characteristic roots $\left(\partial_{i}\right)$ of the fundamental matrix $(\mathrm{A}+\mathrm{B})$ of $(2.2)$ have values $-\delta \leq \partial_{i}<0$, such that the integraldifferential equation of (2.2) at

$t=0$ satisfies the determinant equation, $\operatorname{det}\left(I-B\left(\frac{1-e^{-\partial h_{\max }}}{\partial}\right)\right)=0$.

\section{Proof:}

Assume (2.1) to be of the form

$\frac{d}{d t} F(t)=(A+B) F(t), \quad F(0)=k$

Let us choose a constant non singular matrix $\mathrm{T}$, such that the transformation

$T^{-1}(A+B) T=D$,

where $D$ is an upper triangular matrix. By change of variable, we defined $F(t)=T y$, so that $(2.7)$ is written as

$$
\frac{d y}{d t}=D y, \quad y(0)=k^{\prime}
$$

$k$ and $k^{\prime}$ are constant real values. System (2.9) has a matrix form;

$$
\left[\begin{array}{l}
\dot{y}_{1}(t) \\
\dot{y}_{2}(t) \\
\dot{y}_{3}(t) \\
\cdot \\
\cdot \\
\cdot \\
\dot{y}_{n}(t)
\end{array}\right]=\left[\begin{array}{lllll}
\partial_{11} & \partial_{12} & \partial_{13} & \ldots & \partial_{1 n} \\
& \partial_{22} & \partial_{23} & \ldots & \partial_{2 n} \\
& \partial_{33} & \ldots & \partial_{3 n} \\
& & & & \\
& & & & \\
y_{21} \\
\cdot \\
\cdot \\
\cdot \\
y_{n 1}
\end{array}\right],\left[\begin{array}{l}
y_{11} \\
y_{31} \\
\cdot \\
\dot{y}_{n 1}^{(0)}
\end{array}\right],\left[\begin{array}{l}
\dot{y}_{11}^{(0)} \\
\dot{y}_{21}^{(0)} \\
\dot{y}_{31}^{(0)} \\
\cdot \\
\cdot \\
k_{n 1}^{\prime}
\end{array}\right]=\left[\begin{array}{l}
k_{11}^{\prime} \\
k_{21}^{\prime} \\
k_{31} \\
\cdot \\
\cdot \\
\cdot \\
\\
\end{array}\right.
$$

where $\partial_{i j}$, for $i=j$ are the characteristic roots (Gourlay and Watson, 1973). Assume $\operatorname{Re}\left(\partial_{i i}\right)<0, \quad i=1,2, \ldots . n$, then

$$
y_{n}=k_{n} e^{\partial_{n n} t}
$$

and $y_{n} \rightarrow 0$, as $t \rightarrow \infty$. This implies asymptotic stability Hale(1977). If $\operatorname{Re}\left(\partial_{i i}\right)>0$, then $y_{n} \rightarrow \infty$, as $t \rightarrow \infty$, which contradicts the concept of stability .

Consider the difference-integral of (2.1) defined as

$$
\varphi(t)-B \int_{t-h}^{t} \varphi(s) d s=0, \quad t \geq 0 .
$$


Assume $t=0$, then (2.11) implies

$$
\varphi(0)-B \int_{-h}^{0} e^{\partial_{i i} s} d s=0
$$

Integrating the equation, we obtain

$$
\left(I-B\left(\frac{e^{\partial_{i i} s}}{\partial_{i i}}\right)_{-h_{\max }}^{0}\right)=0
$$

which implies

$$
\left(I-B\left(\frac{1-e^{-\partial_{i i} h \max }}{\partial_{i i}}\right)\right)=0 \quad \partial_{i i}<0 .
$$

Hence

$$
\operatorname{det}\left(I-B\left(\frac{1-e^{-\partial i h m a x}}{\partial_{i i}}\right)\right)=0
$$

\subsection{NUMERICAL APPLICATION.}

1. Consider the retarded differential system,

$$
\dot{x}(t)=A x(t)+B x(t-h)
$$

where

$$
A=\left(\begin{array}{rr}
-1 & 1 \\
1 & -2
\end{array}\right), \quad B=\left(\begin{array}{rr}
0 & -1 \\
-1 & 0
\end{array}\right), \quad C=(A+B)=\left(\begin{array}{rr}
-1 & 0 \\
0 & -2
\end{array}\right)
$$

Assume matrix $Q$ is an identity matrix, so that the quadratic matrix equation is of the form ( $A$ $+B)^{\top} P+P(A+B)=-I$. Resolving the quadratic matrix equation for $P$ being positive symmetric as follow;

$$
\left(\begin{array}{lcc}
2 c_{11} & 2 c_{21} & 0 \\
c_{12} & \left(c_{11}+c_{22}\right) & c_{21} \\
0 & 2 c_{12} & 2 c_{22}
\end{array}\right)\left(\begin{array}{l}
p_{11} \\
p_{12} \\
p_{22}
\end{array}\right)=\left(\begin{array}{c}
-1 \\
0 \\
-1
\end{array}\right),
$$

and substituting the values of $c_{11}, c_{12}, c_{22}$ from matrix $C$, the positive symmetric matrix $P$ is obtained as

$$
P=\left(\begin{array}{ll}
2 & 0 \\
0 & 2
\end{array}\right) \text {, and }|P|>0 \text {. }
$$

Equation (2.5) and (2.6) is satisfied, for any appropriate choice of a symmetric matrix W, with its diagonal element $a_{i i}=1$ or zero(0) and $0 \leq w_{i} \leq 1$, where $w_{i}$ define the $i^{\text {th }}$ row of $W$. Assume $W=\left(\begin{array}{ll}1 & 0 \\ 0 & 1\end{array}\right)$, and recalling (2.4) for $\quad \sum_{11}=\left|-Q+B W^{T} P^{T}\right|, \quad \sum_{22}=|W B|, \quad \sum_{33}=\left|P B(A+B)^{T}\right|$, where $A$ and $B$ are as in (3.1), $P$ as computed in (3.2) and $Q=I$ as stated. Then

$$
\sum_{11}=-3, \quad \sum_{22}=-1, \quad \sum_{33}=-8
$$

and therefore the symmetric matrix $\sum_{i i} \leq 0$, showing system (3.1) is asymptotically stable (see theorem 1, section 2.0) .

Also by theorem 2 the fundamental matrix $C$ has negative real roots $\left(\partial_{1}=-1, \partial_{2}=-2\right)$. Therefore the maximum time delay $\left(h_{\max }\right)$ for (3.1) to attain asymptotic stability is computed as

$$
\operatorname{det}\left(I-B\left(\frac{1-e^{-\partial_{i i} h \max }}{\partial_{i i}}\right)\right)=0
$$

That is for $\partial_{1}=-2$; 


$$
\begin{aligned}
& \operatorname{det}\left(\left(\begin{array}{ll}
1 & 0 \\
0 & 1
\end{array}\right)-\left(\begin{array}{rr}
0 & -1 \\
-1 & 0
\end{array}\right)\left(\frac{1-e^{-2 h \max }}{-2}\right)\right)=0 \\
& \left|\begin{array}{cc}
1 & \frac{1-e^{-2 h \max }}{-2} \\
\frac{1-e^{-2 h \max }}{-2} & 1
\end{array}\right|=0 \\
& 1-\left(1 / 4\left(1-e^{-2 h \max ^{2}}\right)=0\right. \\
& 3-2 e^{-2 h \max }-e^{-4 h \max }=0 .
\end{aligned}
$$

Solving for $h_{\max }$, using the transformation $3-2 y-y^{2}=0, h_{\max }=0.56$.

$$
\begin{aligned}
& \text { For } \partial_{2}=-1 ; \\
& 1-\left(1-e^{-h \max }\right)^{2}=0 .
\end{aligned}
$$

The maximum delay interval is not defined.

Therefore, the maximum delay interval for (3.1) to assume asymptotic stability is $h_{\max }=0.56$.

2. Consider the time-delay system

$$
\left.\begin{array}{l}
\dot{x}_{1}(t)=-3 x_{1}(t)-5 / 2 x_{2}(t)+q x_{2}(t-h) \\
\dot{x}_{2}(t)=-x_{1}(t)+1 / 2 x_{2}(t)+q x_{1}(t-h) .
\end{array}\right\}
$$

System (3.4) has a general form as

$$
\dot{x}(t)=A x(t)+B x(t-h)
$$

where

$$
A=\left(\begin{array}{cc}
-3 & -5 / 2 \\
1 & 1 / 2
\end{array}\right), \quad B=\left(\begin{array}{ll}
0 & q \\
q & 0
\end{array}\right)
$$

and

$$
C=(A+B)=\left(\begin{array}{ll}
-3 & -5 / 2+q \\
1+q & 1 / 2
\end{array}\right) \text {. }
$$

Assume matrix $Q$ is an identity matrix, so that the quadratic matrix equation $(A+B)^{\top} P+$ $P(A+B)=-I$. The quadratic matrix equation for $P$ being symmetric is resolved as,

$$
\left(\begin{array}{lcc}
2 c_{11} & 2 c_{21} & 0 \\
c_{12} & \left(c_{11}+c_{22}\right) & c_{21} \\
0 & 2 c_{12} & 2 c_{22}
\end{array}\right)\left(\begin{array}{l}
p_{11} \\
p_{12} \\
p_{22}
\end{array}\right)=\left(\begin{array}{c}
-1 \\
0 \\
-1
\end{array}\right) .
$$

Substituting the values of $c_{11}, c_{12}, c_{22}$ from (3.5), the positive symmetric matrix $P$ is obtained as

$$
P=\frac{1}{6(1+2 q(1+q))}\left(\begin{array}{cc}
1+2 q(1+q) & q(1+q) \\
q(1+q) & -3
\end{array}\right) \text {. }
$$

The matrix $P$, for all real values of $q$ is not symmetric positive, and therefore does not satisfy theorem 1. Also, all real roots of the fundamental matrix (3.5) for all real values of $q$ is not negative as stated in theorem 2. Therefore the solution of system (3.4) cannot attain asymptotic stability.

\subsection{CONCLUSION}

The integral-differential equation and the positive symmetric properties of given matrices have been used to formulate Lyapunov functional. The introduction of a convex set segment of a symmetric matrix is utilized to establish the boundedness of the first derivative of the formulated functional which satisfies the Lyapunov Krasvoskii condition for asymptotic stability. The integraldifferential equation is also explored to compute the maximum delay interval $\left(h_{\max }\right)$ at which the system solution assumed asymptotic stability. The results obtained show that system (3.1) attained asymptotic stability at $h_{\max }=0.56$, and system (3.4) has no stable solution. 


\section{REFERENCES}

Asl, F. M. and Ulsoy, A. G., 2003. Analysis of System of Linear Delay Differential Equations, Journal of Dynamic Systems, Measurement and Control, (125): 215-223.

Cao, Y. Y., Zongli, L. and Tingshu, H., 2002. Stability Analysis of Linear Time-Delay Systems Subject to Input Saturation, IEE Transactions on Circuit and Systems. Fundamental Theory and Application, (49) 2: 233-240.

Davies, I., 2006. Criteria for Stability and Boundeness of Linear Delay System, Journal of the Mathematical Association of Nigeria, (33) 2B: 419-426.

Driver, R. D., 1977. Ordinary and Delay Differential Equations, Springer - Verlag, New York.

Guglielmi, N. and Hairer, E., 2007. Stiff Delay Equations, Scholarpedia, 2(11) no 2, 2850.

Gourlay, A. R. and Watson, G. A., 1973. Computational Methods for Matrix Eigen problems, John Willy \& Sons. New York.

Hale, J. K., 1977. Theory of Differential Equation, Springer - Verlag, New York.

Hale, J. K. and Verduyn L., S. M., 1993. Introduction to Functional Differential Equation, Springer - Verlag, New York.

Han, Q. L., 2001. On Delay - Dependent Stability for Neutral Delay Differential Systems, (11)4: 965-976.

Kartsatos, G. A., 1980. Ordinary Differential Equations, Mariner Publishing Company Inc, Tumpa, Florida.

Liu, X. Y. and Mansour, M., 1984. Stability Test and Stability Conditions for Delay Differential Systems, Int. J. Control, (39) 6: 1229 - 1242.

Svoboda, Z., 2005. Positive Solutions of P-type Retarded Functional Linear Differential Equations, Proceedings of Equadiff, (11): 475-484. 